\title{
Liver Transection with Clamp Crushing, Followed by Vessel Sealing, is Superior to Crushing by LigaSure in Terms of Early Local Recurrence after Hepatectomy: A Retrospective Cohort Study
}

Kyoji Ito, Nobuyuki Takemura, Fuyuki Inagaki, Fuminori Mihara, Toshiaki Kurokawa, Yoshihiro Edamoto, and Norihiro Kokudo

Hepato-Biliary-Pancreatic Surgery Division, Department of Surgery, National Center for Global Health and Medicine, 1-21-1 Toyama, Shinjuku-ku, Tokyo 162-8655, Japan

\section{ABSTRACT}

Background: Liver transection consists of two elements, crushing of the liver parenchyma and sealing of the remaining tissue. We aimed to investigate the influence of the technique used for crushing liver parenchyma on clinical outcomes in patients with hepatocellular carcinoma (HCC).

Methods: We investigated data from 65 consecutive patients who underwent open hepatectomy for HCC between January 2012 and December 2018. Patients were divided into two groups based on the technique used for crushing liver parenchyma, defined as the direct LigaSure crushing group (DLC group) and clamp-crushing group (CC group).

Results: The direct LigaSure crushing method was associated with a higher rate of early local recurrence than the clamp-crushing method (DLC group vs. CC group, $31.6 \%$ vs. $11.1 \%, p=0.46$ ). A multivariable analysis revealed that estimated blood loss, liver transection method, number of tumors, and curative resection were independent risk factors associated with early local recurrence.

Conclusions: The clamp crushing method may reduce early local recurrence compared with the direct LigaSure crushing method after hepatectomy in HCC patients.

Key words: hepatocellular carcinoma, liver transection, clamp crushing method, LigaSure, thermal injury, early local recurrence

\section{INTRODUCTION}

Liver transection techniques have been a topic of debate for decades, especially in hepatectomy for hepatocellular carcinoma (HCC), because intraoperative bleeding and perioperative blood transfusion are associated with a higher risk of morbidity, mortality, recurrence free survival, and overall survival (1-5). The clamp-crushing method in which the liver parenchyma is clamped, typically with Pean or Kelly forceps, has been a feasible and useful method for liver parenchyma transection, $(6,7)$. and has been reported to be effective in controlling hemorrhage and securing wider tumor-free margins (8). Following

\author{
Corresponding author: \\ Norihiro Kokudo, MD \\ Department of Surgery \\ National Centre for Global Health and \\ Medicine, 1-21-1 Toyama, Shinjuku-ku \\ Tokyo 162-8655, Japan \\ Tel: +81-3-3202-7800 \\ Fax: +81-3-5273-0113 \\ E-mail: nkokudo@hosp.ncgm.go.jp
}

\section{Abbreviations:}

CC - clamp crushing;

DLC - direct LigaSure crushing;

HCC - hepatocellular carcinoma;

ICG R15 - indocyanine green retention rate at 15 minutes.

Received: 23.02.2020

Accepted: 25.04.2020

Copyright (1) Celsius Publishing House www.sgo-iasgo.com 
clamp crushing of the liver parenchyma, the remaining vascular and biliary structures were originally ligated in the clamp-crushing method. However, recent reports revealed that the sealing of the remaining tissues using an electric device including ultrasonic dissection or bipolar device demonstrated equal or better clinical outcomes compared with ligation, in terms of blood loss, operative time and postoperative complication (9-12). Importantly, liver transection techniques could be divided into the "crushing" and "sealing" methods.

Advancement of laparoscopic instruments and surgical techniques over the last two decades has enabled laparoscopic hepatectomy. Specifically, a vessel sealing system using bipolar electrothermal energy (LigaSure, Valleylab, Boulder, CO), which can seal and completely occlude the vessel by denaturing the collagen and elastin in the vessel wall [13)., was widely used in laparoscopic hepatectomy $(14,15)$. In laparoscopic surgery, the liver parenchyma is sometimes directly crushed using the LigaSure jaws because it is difficult to mimic the open 'clamp crush' technique laparoscopically due to limitations in movement of the instruments, and difficulty with frequent switching of instruments during the procedure (15). However, little is known about the difference in clinical outcomes between direct LigaSure crushing and clamp crushing in crushing liver parenchyma.

The aim of the present study was to investigate the influence of liver parenchymal crushing methods on clinical outcomes especially about early local recurrence after hepatectomy in patients with HCC.

\section{METHODS}

\section{Patients}

Between January 2012 and December 2018, 71 consecutive patients underwent hepatectomy for HCC at the National Centre for Global Health and Medicine. Six patients who underwent hepatectomy via a laparoscopic approach were excluded from the study. The remaining 65 patients ( 51 male and 14 female) were included in the analysis, and data were retrieved from prospectively maintained databases including baseline patient characteristics, operative characteristics, and postoperative outcomes. The local institutional review board approved this study (ID: NCGM-G-003206-00). The study was conducted in accordance with the Declaration of Helsinki 1975, as revised in 1983. Informed consent was obtained in the form of an opt-out request on the hospital website.

\section{Indications of surgery}

The indication criteria for liver resection of HCC is described elsewhere [16).. Briefly, liver resection was indicated if liver cirrhosis was categorized into Child-Pugh classification A or B, and the number of tumors was less than four (16). The type of operative procedure used was determined based on the indocyanine green retention rate at 15 minutes (ICG R15) as follows: ICG R15 0-9\%, up to trisegmentectomy; ICG R15 10-19\%, up to bisegmentectomy; ICG R15 20-29\%, up to subsegmentectomy; ICG R15 >30\%, limited resection (17).

\section{Liver transection procedure}

We divided the patients into the direct LigaSure crushing group (DLC group, $n=38$ ) and clamp-crushing group (CC group, $n=27$ ). Three senior surgeons (Y.E., N.T., and N.K.) supervised all operations in this study. Y.E. used the direct LigaSure crushing method, and N.T. and N.K. applied the clamp-crushing method, respectively. The direct LigaSure crushing method was used until June 2017, and the clamp-crushing method after July 2017. After sufficient mobilization of the liver for hepatectomy, transection was performed using an intermittent Pringle's maneuver, with periods of 15 minutes of clamping and 5 minutes of unclamping. In the DLC group, LigaSure was used to divide the parenchyma (15). Briefly, one of the end blades of LigaSure was inserted carefully into the liver parenchyma and the blades were closed softly. Then, the liver parenchyma was crushed and sealed simultaneously by activation of the LigaSure, and the sealed tissue was divided (figure 1). In the CC group, the liver parenchyma was crushed using forceps, and the remaining isolated vessels was sealed and cut with LigaSure (figure 1) (9). The Glissonian sheath, and hepatic veins with a diameter of $3 \mathrm{~mm}$, were ligated in both groups. In both groups, Small Jaw LigaSure was used, and the power of the LigaSure system was set at level 2 . We divided the liver with a tumor-free margin of at least $5 \mathrm{~mm}$ to avoid exposure of the tumor, and anatomical liver resection was basically performed if the future remnant liver volume was sufficient (17). When it was impossible to avoid tumor exposure, liver parenchymal transection was performed along the surface of $\mathrm{HCC}$, with a recorded tumor-free margin of $0 \mathrm{~mm}$. 


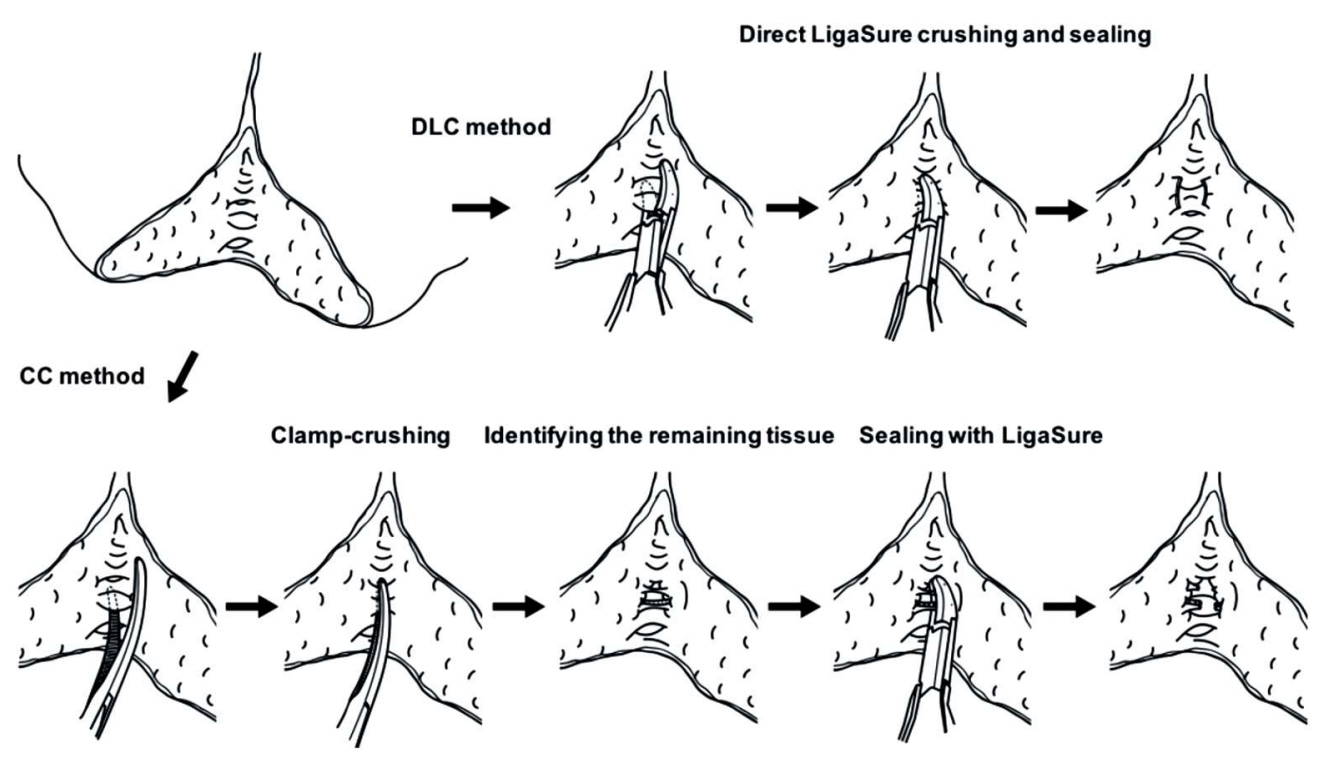

Figure 1 - Liver transection method in the DLC and CC groups. CC, clamp crushing; DLC, direct LigaSure crushing

\section{Follow-up}

After discharge, all patients were examined for HCC recurrence by tumor markers ( $\alpha$-fetoprotein and protein induced by vitamin $\mathrm{K}$ absence or antagonist-II) and ultrasonography every 3 months, and by dynamic computed tomography every 6 months. Dynamic computed tomography was also performed when the tumor markers elevated. Recurrence was defined as the appearance of a new lesion with radiologic features typical of HCC. Early recurrence was defined as occurring within one year after hepatectomy. The recurrence data was obtained at January 31st 2020, and therefore, the follow-up period was at least two years.

\section{Statistical analyses}

Continuous variables were expressed as the median (interquartile ranges) and compared using the Wilcoxon rank-sum test. Categorical variables were expressed as percentages and were compared using the Fisher's exact test or the chi-square test as appropriate. Multivariable analysis using the Cox proportional hazards model was performed to identify predictive factors for the early local recurrence of HCC after hepatectomy, and factors with $p<0.05$ in the univariable analyses were used in the multivariable analysis. Operative time and estimated blood loss were categorized by their median values. A $p$ value $<0.05$ was considered statistically significant. Statistical analyses were performed using JMP software version 9.0.2 (SAS Institute Inc., Cary, NC, USA).

\section{RESULTS}

\section{Patient demographics}

The median age of all patients was 72 (interquartile range: 66-78) years. The baseline patient characteristics and pathological findings of the resected tumors were compared between the DLC and CC groups (table 1). Tumor-free margin was significantly narrower in the CC group than DLC group. The other baseline patient characteristics and other pathological findings were not significantly different between groups.

\section{Surgical outcome}

The surgical outcomes were compared between the DLC and CC groups (table 2). The operative time was significantly longer in the DLC group than the CC group. The length of hospitalization was significantly shorter in the DLC group than the CC group due to the difference of chief surgeon's policy. The rate of the whole early recurrence was not different between groups, while the rate of local recurrence was significantly higher in the DLC group than the CC group (table 1, figure 2). In the DLC group, the tumor-free margin was significantly narrower in patients with early local recurrence than those without $(2.8 \pm 5.5 \mathrm{~mm}$ vs. $6.5 \pm 1.5 \mathrm{~mm}$, $p=0.046)$. There was one case in which hepatectomy 
Table 1 - Baseline patient characteristics and pathological findings of the resected tumor between the groups

\begin{tabular}{|c|c|c|c|}
\hline Variables & $\begin{array}{l}\text { DLC group } \\
(\mathrm{n}=\mathbf{3 8})\end{array}$ & $\begin{array}{c}\text { CC group } \\
(\mathrm{n}=27)\end{array}$ & $P$ value \\
\hline Men/Women & $30 / 8$ & $21 / 6$ & 0.910 \\
\hline Age (years) & $75(65-78)$ & $70(67-77)$ & 0.670 \\
\hline $\mathrm{BMI}\left(\mathrm{kg} / \mathrm{m}^{2}\right)$ & $23.4(20.0-25.0)$ & $24.1(22.1-25.6)$ & 0.239 \\
\hline $\begin{array}{l}\text { Comorbidities } \\
\text { Hypertension, n (\%) } \\
\text { Diabetes mellitus, n (\%) } \\
\text { Cardiac disease, n (\%) } \\
\text { Pulmonary disease, n (\%) }\end{array}$ & $\begin{array}{c}11(31.6) \\
12(31.6) \\
7(18.4) \\
1(2.6)\end{array}$ & $\begin{array}{c}14(51.9) \\
13(48.2) \\
1(3.7) \\
1(3.7)\end{array}$ & $\begin{array}{l}0.073 \\
0.176 \\
0.075 \\
0.805\end{array}$ \\
\hline ASA status $\geq 3, n(\%)$ & $6(15.8)$ & $8(29.6)$ & 0.181 \\
\hline $\begin{array}{l}\text { Cause of cirrhosis, n (\%) } \\
\text { HBV } \\
\text { HCV } \\
\text { NASH } \\
\text { Others }\end{array}$ & $\begin{array}{c}3(7.9) \\
8(21.1) \\
4(10.5) \\
23(60.5)\end{array}$ & $\begin{array}{c}6(22.2) \\
10(37.0) \\
2(7.4) \\
9(33.3)\end{array}$ & 0.116 \\
\hline ICG-R15 (\%) & $11.9(8.6-14.9)$ & $11.8(8.0-19.0)$ & 0.527 \\
\hline Child Pugh Classification $\geq B$ & $3(7.9)$ & $2(7.4)$ & $>.999$ \\
\hline $\mathrm{AFP} \geq 150 \mathrm{ng} / \mathrm{mL}, \mathrm{n}(\%)$ & $6(15.8)$ & $8(29.6)$ & 0.181 \\
\hline PIVKAII, $\geq 100 \mathrm{mAU} / \mathrm{mL}, \mathrm{n}(\%)$ & $17(44.7)$ & $13(48.2)$ & 0.786 \\
\hline Type of resection, major/minor & $13 / 25$ & $9 / 18$ & 0.941 \\
\hline $\begin{array}{l}\text { Pathological characteristics } \\
\text { Tumor size, }(\mathrm{cm}) \\
\text { Number of tumors, } 1 / 2 />3 \\
\text { Tumor-free margin, (mm) } \\
\text { Curative resection, } \mathrm{n}(\%)\end{array}$ & $\begin{array}{c}3.9(2.3-8.0) \\
32 / 3 / 3 \\
3.0(1.0-6.0) \\
32(84.2)\end{array}$ & $\begin{array}{c}3.0(2.0-7.5) \\
22 / 3 / 2 \\
1.0(0-4.0) \\
25(92.6)\end{array}$ & $\begin{array}{l}0.383 \\
0.907 \\
0.028 \\
0.311\end{array}$ \\
\hline
\end{tabular}

Abbreviations: ASA, the American Society of Anesthesiologists Physical Status classification system; BMI, body mass index; CC, clamp crushing; HBV, hepatitis B virus; HCV, hepatitis C virus; ICG, indocyanine green; DLC, direct LigaSure crushing; NASH, nonalcoholic steato-hepatitis

was performed along the surface of the HCC using the direct LigaSure crushing method, and pathological analysis indicated potential tumor dissemination caused by cauterization of the surface of the tumor (figure 3).

\section{Risk factors associated with early local recurrence}

Risk factors associated with early local recurrence of HCC after hepatectomy within a year were analyzed
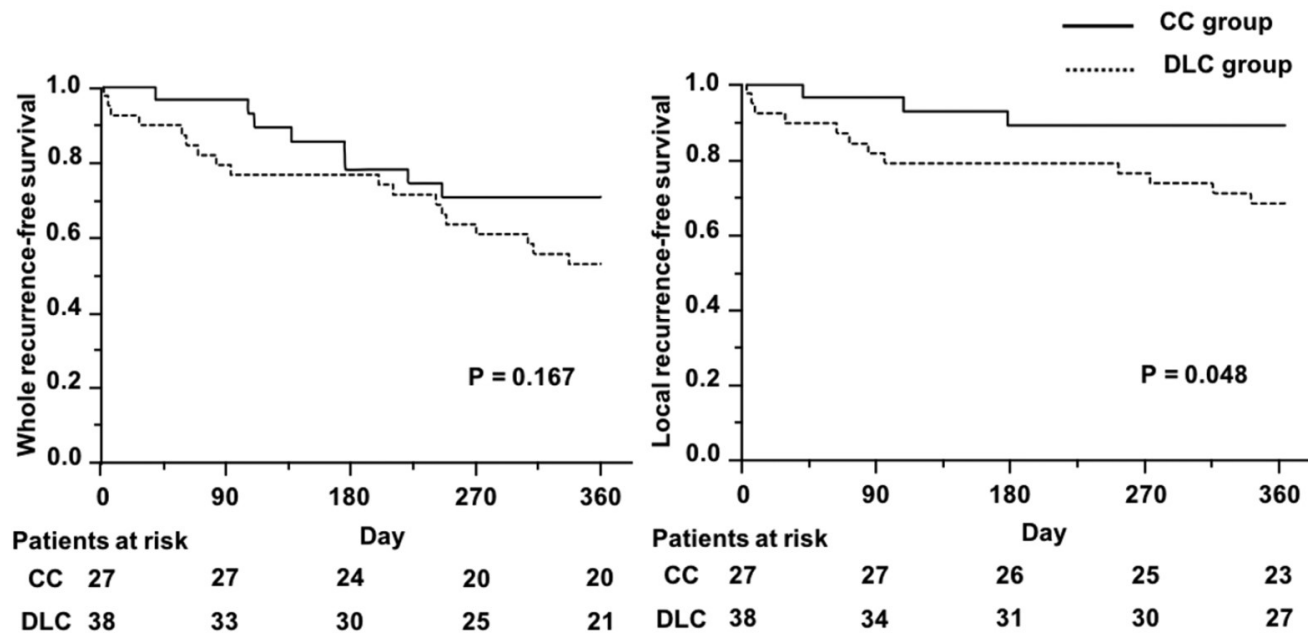

Figure 2 - Whole recurrence-free survival (left) and local recurrence-free survival (right) between the DLC and CC groups. CC, clamp crushing; DLC, direct LigaSure crushing 
Figure 3 - A specimen after anterior segmentectomy using the direct LigaSure crushing method.

(a) macroscopic image of the transection surface. (b) Hematoxylin-eosin staining of the tumor-free margin positive point with cauterization by LigaSure. Denaturation by cauterization of the tumor surface was observed, and tumor cell dissemination was suspected from the surface of the tumor (arrow) a

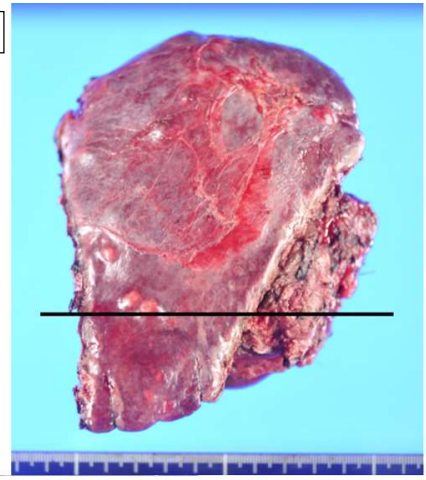

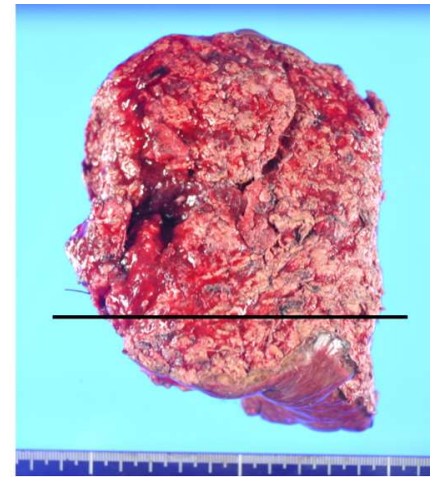
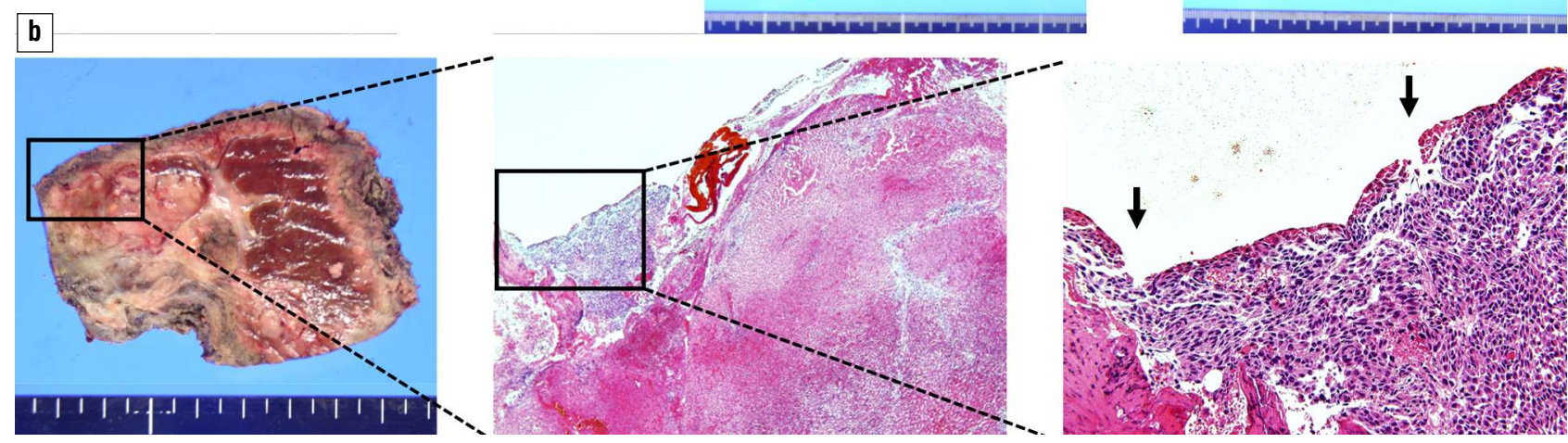

using the Cox proportional hazards model (table 2). Univariable analyses showed that operation time, estimated blood loss, liver transection method, number of tumors, and curative resection were significantly associated with early local recurrence of HCC after hepatectomy. Subsequent multivariable analysis revealed that estimated blood loss $(\mathrm{HR} 5.30,95 \% \mathrm{Cl}$ 1.03-43,54, $\mathrm{P}=0.046$ ), liver transection method (HR 3.66, $95 \% \mathrm{Cl} 1.11-16,71, \mathrm{P}=0.032$ ), number of tumors (HR 3.95, 95\% Cl 1.23-11,98, $\mathrm{P}=0.022$ ), and curative resection ( $\mathrm{HR} 3.27,95 \% \mathrm{Cl} 1.08-9.83, \mathrm{P}=0.037$ ) were independent risk factors associated with early local recurrence (table 2).

\section{DISCUSSION}

The present study demonstrated that liver transection method was associated with a higher rate of early local recurrence in addition to estimated blood loss, number of tumors, and curative resection. In DLC group,

Table 2 - Comparison of intra and postoperative outcomes between the groups

\begin{tabular}{|c|c|c|c|}
\hline Variables & $\begin{array}{l}\text { DLC group } \\
(\mathrm{n}=38)\end{array}$ & $\begin{array}{c}\text { CC group } \\
(\mathrm{n}=27)\end{array}$ & $P$ value \\
\hline $\begin{array}{l}\text { Intraoperative outcomes } \\
\text { Operation time, (min) } \\
\text { Estimated blood loss, (mL) } \\
\text { Blood transfusion, (\%) }\end{array}$ & $\begin{array}{c}350(262-480) \\
235(118-645) \\
2(5.3)\end{array}$ & $\begin{array}{c}293(218-372) \\
440(208-882) \\
6(22.2)\end{array}$ & $\begin{array}{l}0.026 \\
0.058 \\
0.058\end{array}$ \\
\hline $\begin{array}{l}\text { Postoperative outcomes } \\
\text { Major complications, (\%) } \\
\text { Length of hospitalization (days) }\end{array}$ & $\begin{array}{c}2(5.3) \\
10(9-12)\end{array}$ & $\begin{array}{c}2(7.4) \\
15(10-19)\end{array}$ & $\begin{array}{l}>.999 \\
<.001 \\
\end{array}$ \\
\hline Early recurrence, $(\%)$ & $18(47.4)$ & $8(29.6)$ & 0.150 \\
\hline Local recurrence & $12(31.6)$ & $3(11.1)$ & 0.046 \\
\hline Intrahepatic metastasis & $3(7.9)$ & $3(11.1)$ & 0.686 \\
\hline Bone metastasis & $2(5.3)$ & 0 & 0.507 \\
\hline Lung metastasis & $1(2.6)$ & $1(3.7)$ & $>.999$ \\
\hline Peritoneal dissemination & 0 & $1(3.7)$ & 0.415 \\
\hline
\end{tabular}

Abbreviations: CC - clamp crushing; DLC - direct LigaSure crushing 
the tumor-free margin was significantly narrower in patients with early local recurrence than those without, suggesting that thermal injury on the surface of the tumor might affect potential tumor dissemination and subsequent local recurrence after hepatectomy.

Several studies, including randomized controlled trials, have compared postoperative complications and clinical outcomes between the standard clamp crushing and LigaSure in open hepatectomy. Some showed that liver transection using LigaSure reduced transection time, $(9,18)$ although others reported no significant difference between the two methods (10-12). However, these studies compared the methods of sealing the remaining tissue after clamp crushing of liver parenchyma. On the other hand, liver parenchyma might be directly crushed using electrosurgical devises in laparoscopic surgery due to the difficulty in mimicking the open 'clamp crush' technique laparoscopically due to the movement limitations of the instruments $(14,15)$. Liu et al. reported the efficacy of direct LigaSure crushing in terms of shorter transection time and lower costs in laparoscopic hepatectomy compared with the Cavitron Ultrasonic Surgical Aspirator (15). However, there were few reports demonstrating the differences in liver parenchymal crushing techniques between clamp crushing and direct LigaSure crushing in hepatectomy.

Although electrosurgical devices can reduce the risk of bleeding and simplify the surgical procedure in hepatectomy, there are possible concerns about thermal injury caused by ultrasonic or bipolar energy $(19,20)$. Takayama et al. reported a higher incidence of tumor exposure on the liver transection surface when using electrosurgical devices for liver transection, and the CC method enabled achievement of wider tumorfree margins (8). In the present study, the rate of early local recurrence was significantly higher in DLC group than CC group, especially when the tumor-free margin was short, and direct LigaSure crushing method was an independent risk factor of early local recurrence after open hepatectomy in HCC patients. We also showed a case with exposure of the tumor surface and suspicion of tumor cell dissemination caused by the lateral thermal injury during the liver parenchymal transection, suggesting that a larger tumor-free margin might be recommended when direct LigaSure crushing is applied. On the other hand, clamp crushing of the liver parenchyma enables the operator to identify the remaining isolated vessels before sealing, and therefore, the operator is able to seal and cut through the vessels keeping a safe distance away from the tumor. The operator can also choose ligation of the vessels instead of sealing with LigaSure when the tumor is very close to the transection surface. By ligating the vessels after clamp crushing, thermal injury of the tumor surface can be avoided and wider tumor-free margin can be obtained (8). However, because of the several limitations described below, full discussion about the association of the liver transection method and tumor-free margin with early local recurrence was difficult. Further studies are required to demonstrate the influence of the liver parenchymal crushing method and tumor-free margin on early local recurrence after $\mathrm{HCC}$ resection.

The present study has several limitations. First of all, it was a retrospective study, and complete adjustments for all confounding variables were not possible despite the use of a multivariable Cox proportional hazard model analysis. Secondly, the choice of liver parenchymal crushing method depended on the supervising surgeons in this study, which might be a critical flaw in discussing the association between surgical procedure and clinical outcomes. Accordingly, prospective and randomized studies would be required to determine the influence of liver parenchymal crushing method on clinical outcomes after hepatectomy. Finally, relatively small number of patients may have weakened the analyses.

\section{CONCLUSIONS}

Application of the clamp crushing method could reduce early recurrence after hepatectomy in HCC patients. Additionally, a wider tumor-free margin should be recommended when using the direct LigaSure crushing method to prevent early recurrence of HCC.

\section{Acknoelwdgement}

The authors would like to thank Ryohei Kuroda and Hideki Miyazaki for assistance with the pathological results.

\section{Ethics approval and consent to participate}

The National Center for Global Health and Medicine Research Ethics Committee/Institutional Review Board approved this study (approval number: NCGM-G003206-00).

\section{Consent for publication}

Informed consent was obtained in the form of optout on the hospital website. 


\section{Competing interests}

The authors declare that they have no competing interests.

\section{Funding}

No funding was obtained from the private or public sector for this research.

\section{Authors' contributions}

$\mathrm{KI}$ and NT contributed to the conception and design of the study. All authors contributed to the acquisition and analysis of data. KI, NT, and NK were a major contributor in writing the manuscript. All authors read and approved the final manuscript.

\section{REFERENCES}

1. Wei AC, Tung-Ping Poon R, Fan ST, Wong J. Risk factors for perioperative morbidity and mortality after extended hepatectomy for hepatocellular carcinoma. Br J Surg. 2003;90:33-41.

2. Taketomi A, Kitagawa D, Itoh S, Harimoto N, Yamashita Y, Gion T et al. Trends in morbidity and mortality after hepatic resection for hepatocellular carcinoma: an institute's experience with 625 patients. J Am Coll Surg. 2007;204:580-587.

3. Yamamoto J, Kosuge T, Takayama T, Shimada K, Yamasaki S, Ozaki $\mathrm{H}$ et al. Perioperative blood transfusion promotes recurrence of hepatocellular carcinoma after hepatectomy. Surgery 1994;115 303-309.

4. Shiba H, Ishida Y, Wakiyama S, lida T, Matsumoto M, Sakamoto T et al. Negative impact of blood transfusion on recurrence and prognosis of hepatocellular carcinoma after hepatic resection. $J$ Gastrointest Surg. 2009;13:1636-1642.

5. Katz SC, Shia J, Liau KH, Gonen M, Ruo L, Jarnagin WR et al. Operative blood loss independently predicts recurrence and survival after resection of hepatocellular carcinoma. Ann Surg. 2009;249: 617-623.

6. Lin T. Results in 107 hepatic lobectomies with a preliminary report on the use of a clamp to reduce blood loss. Ann Surg. 1973; 177: 413 .

7. Lin T-Y. A simplified technique for hepatic resection: the crush method. Ann Surg. 1974;180:285.

8. Takayama T, Makuuchi M, Kubota K, Harihara Y, Hui AM, Sano K et al. Randomized comparison of ultrasonic vs clamp transection of the liver. Arch Surg. 2001:136:922-928.

9. Saiura A, Yamamoto J, Koga R, Sakamoto Y, Kokudo N, Seki M et al. Usefulness of LigaSure for liver resection: analysis by randomized clinical trial. Am J Surg. 2006;192:41-45.

10. Ikeda M, Hasegawa K, Sano K, Imamura H, Beck Y, Sugawara $Y$ et al. The vessel sealing system (LigaSure) in hepatic resection: a randomized controlled trial. Ann Surg. 2009;250:199-203.

11. Garancini M, Gianotti L, Mattavelli I, Romano F, Degrate L, Caprotti $R$ et al. Bipolar vessel sealing system vs. clamp crushing technique for liver parenchyma transection. Hepatogastroenterology. 2011;58: 127-132.

12. Doklestic K, Karamarkovic A, Stefanovic B, Stefanovic B, Milic N, Gregoric $P$ et al. The efficacy of three transection techniques of the liver resection: a randomized clinical trial. Hepatogastroenterology 2012;59:1501-1506

13. Romano F, Franciosi C, Caprotti R, Uggeri F, Uggeri F. Hepatic surgery using the Ligasure vessel sealing system. World J Surg. 2005:29:110-112.

14. Huanwei C, Feiwen D. Pure laparoscopic right hemihepatectomy via anterior approach. Surg Endosc. 2016;30:5621-5621.

15. Liu F, Wei Y, Li H, Wang W, Wen T, Wu H et al. LigaSure versus CUSA for parenchymal transection during laparoscopic hepatectomy in hepatocellular carcinoma patients with cirrhosis: a propensity score-matched analysis. Surg Endosc. 2018;32:2454-2465.

16. Kokudo N, Makuuchi M. Evidence-based clinical practice guidelines for hepatocellular carcinoma in Japan: the J-HCC guidelines. J Gastroenterol. 2009:44:119-121.

17. Makuuchi M, Kosuge T, Takayama T, Yamazaki S, Kakazu T, Miyagawa S et al. Surgery for small liver cancers. Semin Surg Oncol. 1993;298-304.

18. Gotohda N, Yamanaka T, Saiura A, Uesaka K, Hashimoto M, Konishi $\mathrm{M}$ et al. Impact of energy devices during liver parenchymal transection: a multicenter randomized controlled trial. World J Surg. 2015;39:1543-1549.

19. Sutton PA, Awad S, Perkins AC, Lobo DN. Comparison of lateral thermal spread using monopolar and bipolar diathermy, the Harmonic Scalpe ${ }^{\mathrm{TM}}$ and the Ligasure ${ }^{\mathrm{TM}}$. Br J Surg. 2010;97:428-433.

20. Seehofer D, Mogl M, Boas-Knoop S, Unger J, Schirmeier A, Chopra $S$ et al. Safety and efficacy of new integrated bipolar and ultrasonic scissors compared to conventional laparoscopic 5-mm sealing and cutting instruments. Surg Endosc. 2012;26:2541-2549. 